\title{
MARKOV PROCESSES WITH IDENTICAL HITTING PROBABILITIES
}

BY

JOSEPH GLOVER

\begin{abstract}
Let $\left(X(t), P^{x}\right)$ and $\left(Y(t), Q^{x}\right)$ be transient Hunt processes on a state space $E$ satisfying the hypothesis of absolute continuity (Meyer's hypothesis (L)). Let $T(K)$ be the first entrance time into a set $K$, and assume $P^{x}(T(K)<\infty)=$ $Q^{x}(T(K)<\infty)$ for all compact sets $K \subseteq E$. There exists a strictly increasing continuous additive functional of $X(t), A(t)$, so that if $T(t)=\inf \{s: A(s)>t\}$, then $\left(X(T(t)), P^{x}\right)$ and $\left(Y(t), Q^{x}\right)$ have the same joint distributions. An analogous result is stated if $X$ and $Y$ are right processes (with an additional hypothesis). These theorems generalize the Blumenthal-Getoor-McKean Theorem and have interpretations in terms of potential theory.
\end{abstract}

0. Introduction. In 1962, Blumenthal, Getoor and McKean [2] proved the following theorem in a paper entitled Markov processes with identical hitting distributions.

(0.1) ThEOREM. Let $\left(\tilde{X}_{t}, \tilde{P}^{x}\right)$ and $\left(\tilde{Y}_{t}, \tilde{Q}^{x}\right)$ be standard processes on $\left(E_{\Delta}, \mathcal{E}_{\Delta}\right)$ so that for each compact subset $K$ of $E, \tilde{P}_{K}(x, \cdot)=\tilde{Q}_{K}(x, \cdot)$ for all $x$. There exists a continuous additive functional $A_{t}$ of $\tilde{X}_{t}$ which is strictly increasing and finite on $[0, \zeta)$ so that if $T_{t}$ is the right continuous inverse of $A_{t}$, then $\left(\tilde{X}\left(T_{t}\right), \tilde{P}^{x}\right)$ and $\left(\tilde{Y}_{t}, \tilde{Q}^{x}\right)$ have the same joint distributions.

Here $\tilde{P}_{K}(x, \cdot)$ and $\tilde{Q}_{K}(x, \cdot)$ are the distributions of $\tilde{X}$ and $\tilde{Y}$ at $T_{K}$, the first hitting time of $K$. Their proof works equally well if $\tilde{X}$ and $\tilde{Y}$ are right processes (the hypothèses droites were formulated later than 1962). Now this theorem is of interest not only in probability theory, but in potential theory as well. The object of interest in potential theory is the cone of excessive functions associated to the Markov process. Hunt's balayage theorem [1, III-6.12] tells how to construct the hitting operators $\tilde{P}_{K}$ given the cone of excessive functions, and Dynkin's theorem [1, II-5.1] tells how to determine the cone of excessive furctions given the hitting operators. Thus, Theorem ( 0.1$)$ states that if two right processes have the same potential theory (i.e. the same excessive functions), then they are related by a time change as described above. Also, it is simple to observe that time changing $\tilde{X}$ in the fashion described in $(0.1)$ leaves the hitting operators unchanged, so the potential theory does not change.

Received by the editors October 7, 1981.

1980 Mathematics Subject Classification. Primary 60J25; Secondary $31 \mathrm{C} 15$.

Key words and phrases. Transient Hunt process, time change, Blumenthal-Getoor-McKean Theorem, continuous additive functional, réduite.

'Research supported in part by NSF grant MCS-8002659 and CNRS Fellowship while the author was visiting I. M. S. S., Université de Grenoble II. 
Last exit times and distributions have become increasingly important in Markov processes in recent years, and it is natural to ask if last exit distributions characterize a Markov process up to time change in the same way that first entrance distributions characterize the processes in (0.1). The answer is yes if $\tilde{X}$ and $\tilde{Y}$ are transient Hunt processes [7].

(0.2) THeOREM. Let $\left(\tilde{X}_{t}, \tilde{P}^{x}\right)$ and $\left(\tilde{Y}_{t}, \tilde{Q}^{x}\right)$ be two Hunt processes on $\left(E_{\Delta}, \mathcal{E}_{\Delta}\right)$ so that $L_{K}=\sup \left\{t: \tilde{X}_{t} \in K\right\}<\infty$ almost surely and $M_{K}=\sup \left\{t: \tilde{Y}_{t} \in K\right\}<\infty$ almost surely (sup $\varnothing=0)$ for all compact subsets $K$ of $E$. Assume

$$
\tilde{P}^{x}\left(f\left(\tilde{X}_{L_{K^{-}}}\right) ; L_{K}>0\right)=\tilde{Q}^{x}\left(f\left(\tilde{Y}_{M_{K^{-}}}\right) ; M_{k}>0\right)
$$

for all bounded functions $f$ on $E$ and for all compact subsets $K$ of $E$. There exists $a$ strictly increasing continuous additive functional of $\tilde{X}_{t}, A_{t}$, so that if $T_{t}$ is the right continuous inverse of $A_{t}$, then $\left(\tilde{X}\left(T_{t}\right), \tilde{P}^{x}\right)$ and $\left(\tilde{Y}_{t}, \tilde{Q}^{x}\right)$ have the same joint distributions.

It is worth noting that while this seems to be a "dual" theorem to (0.1) (in that the hypothesis of equality of first entrance distributions is replaced with equality of last exit distributions), it does not seem possible to prove $(0.2)$ using $(0.1)$ and a time-reversal argument. It is easy to check that $\tilde{P}^{x}\left(f\left(\tilde{X}_{L_{K^{-}}}\right) ; L_{K}>0\right)$ is an excessive function if $f$ is positive. Under mild additional assumptions (such as classical duality) we can write $\tilde{P}^{x}\left(f\left(\tilde{X}_{L_{K^{-}}}\right) ; L_{K}>0\right)=\int u(x, y) f(y) \pi_{K}(d y)=\tilde{U} f \pi_{K}(x)$, where $u(x, y)$ is the potential density for $\tilde{X}$ and $\pi_{K}$ is the equilibrium measure of $K$. In this case, the hypothesis in (0.2) is equivalent to assuming that $\tilde{U} f \pi_{K}=\tilde{V} f \gamma_{K}$ for all bounded Borel functions $f$ and all compact sets $K$, where $\tilde{V}$ is the potential kernel for $\tilde{Y}$ and $\gamma_{K}$ is the equilibrium measure of $K$ for $\tilde{Y}$. The conclusion we draw from (0.2) is that the potential theories of $\tilde{X}$ and $\tilde{Y}$ are the same.

Now both (0.1) and (0.2) require a lot of information, namely the first entrance or last exit distributions for all compact sets $K$. If only the first entrance probabilities agree $\left(\tilde{P}^{x}\left(T_{K}<\infty\right)=\tilde{Q}^{x}\left(T_{K}<\infty\right)\right.$ for all compact sets $\left.K\right)$, can we draw the same conclusions as in (0.1) and (0.2)? Notice that $\tilde{P}^{x}\left(T_{K}<\infty\right)=\tilde{P}^{x}\left(L_{K}>0\right)$, so we can equally well phrase the hypothesis in terms of last exit probabilities by requiring $\tilde{P}^{x}\left(L_{K}>0\right)=\tilde{Q}^{x}\left(M_{K}>0\right)$.

The following two statements answer the question. Let $\tilde{X}$ (resp. $\tilde{Y}$ ) have resolvent $\left(\tilde{U}^{a}\right)_{a \geqslant 0}$ (resp. $\left.\left(\tilde{V}^{a}\right)_{a \geqslant 0}\right)$.

(0.3) Let $\left(\tilde{X}_{t}, \tilde{P}^{x}\right)$ and $\left(\tilde{Y}_{t}, \tilde{Q}^{x}\right)$ be two transient Hunt processes so that $\tilde{U}^{a}(x, \cdot)$ $\ll \eta$ and $\tilde{V}^{a}(x, \cdot) \ll \rho$ for all $a \geqslant 0$ and for all $x$ in $E$. If $\tilde{P}^{x}\left(T_{K}<\infty\right)=\tilde{Q}^{x}\left(T_{K}<\infty\right)$ for all compact sets $K$ in $E$, then $\tilde{X}$ and $\tilde{Y}$ are related by a time change as in $(0.1)$ and (0.2) (see Theorem (2.1)).

(0.4) Let $\left(\tilde{X}_{t}, \tilde{P}^{x}\right)$ and $\left(\tilde{Y}_{t}, \tilde{Q}^{x}\right)$ be two transient right processes so that $\tilde{U}^{a}(x, \cdot) \ll \eta$ and $\tilde{V}^{a}(x, \cdot) \ll \rho$ for all $a \geqslant 0$ and for all $x$ in $E$. Assume

(0.5) $\tilde{P}^{x}\left(T_{K}<\infty\right)=\tilde{Q}^{x}\left(T_{K}<\infty\right)$ for all compact sets $K$ in $E$.

(0.6) Within each nonpolar set $G \subset E$, there is a nonpolar compact set $L \subset G$ so that $\tilde{P}^{x}\left(T_{L}<\infty\right)=\tilde{U} \pi_{L}(x)$ and $\tilde{Q}^{x}\left(T_{L}<\infty\right)=\tilde{V} \gamma_{L}(x)$, where $\pi_{L}$ and $\gamma_{L}$ are measures supported on $L$. 
Then $\tilde{X}$ and $\tilde{Y}$ are related by a time change as in $(0.1)$ and $(0.2)$ (see Theorem (1.3)).

The function $\tilde{P}^{x}\left(T_{K}<\infty\right)$ is well known in potential theory: it is the réduite of the function 1 on the set $K$. Thus (0.3) may be rephrased as follows: if two cones of excessive functions (corresponding to Hunt processes satisfying the hypothesis of absolute continuity) have the same réduites of 1 on compact sets, then the cones must be the same.

The proofs of $(0.3)$ and (0.4)-(0.6) are, of course, technical, and therefore it may be of some use to examine the simplest case of $(0.3)$ where $\tilde{X}$ and $\tilde{Y}$ are transient Hunt processes on a finite state space $E=\{1,2,3, \ldots, n\}$. In this case, it is well known that there are measures $\mu$ and $\nu$ on $E$ and Hunt processes $\tilde{Z}$ and $\tilde{W}$ so that $\tilde{X}$ and $\tilde{Z}$ are in classical duality with respect to $\mu$ and $\tilde{Y}$ and $\tilde{W}$ are in classical duality with respect to $\nu$ (see [1, Chapter VI] for a discussion of duality). Then $\tilde{P}^{x}\left(T_{K}<\infty\right)$ $=\tilde{P}^{x}\left(L_{K}>0\right)=\tilde{U} \pi_{K}(x)$ and $\tilde{Q}^{x}\left(T_{K}<\infty\right)=\tilde{Q}^{x}\left(M_{K}>0\right)=\tilde{V} \gamma_{K}(x)$. Taking $K=$ $\{y\}$, we observe that $\pi_{K}(d z)=c(y) \varepsilon_{\{y\}}(d z)$ and $\gamma_{K}(d z)=d(y) \varepsilon_{\{y\}}(d z)$, where $c(y)>0$ and $d(y)>0$. Thus $u(x, y) c(y)=v(x, y) d(y)$. Let $m(y)=\mu(\{y\})$, and let $r(y)=\nu(\{y\})$. If we set $A_{t}=\int_{0}^{t}(c r)\left(\tilde{X}_{s}\right) /(d m)\left(\tilde{X}_{s}\right) d s$ and $T_{t}=\inf \left\{s: A_{s}>t\right\}$, then

$$
\begin{aligned}
\tilde{P}^{x} \int f\left(\tilde{X}\left(T_{t}\right)\right) d t & =\tilde{P}^{x} \int f\left(\tilde{X}_{t}\right) d A_{t}=\int u(x, y) f(y)(c r)(y) /(d m)(y) \mu(d y) \\
& =\int v(x, y) f(y) \nu(d y)=\tilde{Q}^{x} \int f\left(\tilde{Y}_{t}\right) d t
\end{aligned}
$$

It follows from [1, V-5.10] that $\left(\tilde{X}\left(T_{t}\right), \tilde{P}^{x}\right)$ has the same distribution as $\left(\tilde{Y}_{t}, \tilde{Q}^{x}\right)$.

Finally, we remark that the well-known theorem stating that any one-dimensional conservative regular diffusion on $(0,1)$ can be transformed (by a change of scale and time) into a Brownian motion is a corollary of (0.3).

Precise definitions are given in the next section. We shall use standard notation found in [1 and 4]. If $K$ is a subset of a compact metric space, then $\mathbf{C}(K)$ (resp. $\left.b \mathbf{C}(K)^{+}\right)$denotes the collection of restrictions to $K$ of all continuous functions on the metric space (resp. which are bounded and positive). Sigma fields and the collection of functions measurable over them will be denoted in the same way. Thus, $\mathscr{F}$ may be the collection of sets in $\mathscr{F}$, and $b \mathscr{F}$ (resp. $\mathscr{F}^{+}, b \mathscr{F}^{+}$) is the collection of bounded $\mathscr{F}$-measurable functions (resp. positive $\mathscr{F}$-measurable functions, bounded and positive $\mathscr{F}$-measurable functions).

1. The basic time change theorem for right processes. Let $\tilde{X}=\left(\Omega, \tilde{\mathscr{F}}, \tilde{\mathscr{F}}_{t}, \tilde{X}_{t}, \tilde{\theta}_{t}, \tilde{P}^{x}\right)$ be a right process on a Lusin topological space $E_{\Delta}$ together with its Borel field $\mathcal{E}_{\Delta}$ [4]. Let $\tilde{P}_{t}$ be the semigroup of $\tilde{X}$, and let $\left(\tilde{U}^{a}\right)_{a>0}$ be the resolvent of $\tilde{X}$. As usual, we assume that $\Delta$ acts as a trap for $\tilde{X}$. When we refer to a "Borel function $f$ on $E$ ", $f$ is assumed to be zero at $\Delta$, and the resolvent is not considered to charge $\Delta$. We make two other assumptions which will hold throughout the paper. First, $\tilde{X}$ is transient: there is a Borel function $h^{X}$ bounded by 1 which is strictly positive on $E=E_{\Delta}-\{\Delta\}$ so that $\tilde{U} h^{X} \leqslant 1$ on $E_{\Delta}$. Second, we assume $\eta$ is a reference probability measure on $E$ 
for $\tilde{X}$ so that $\tilde{U}^{a}(x, \cdot) \ll \eta$ for all $x$ in $E$ and for all nonnegative $a$. Because of this assumption, $\tilde{P}_{t}$ is Borel measurable.

Let $\tilde{Y}=\left(\Omega, \tilde{\mathcal{S}}_{,}, \tilde{\mathcal{S}}_{t}, \tilde{Y}_{t}, \tilde{\theta}_{t}, \tilde{Q}^{x}\right)$ be another right process on $\left(E_{\Delta}, \varepsilon_{\Delta}\right)$ with semigroup $\tilde{Q}_{t}$ and resolvent $\left(\tilde{V}^{a}\right)_{a>0}$. We assume that $\tilde{Y}$ is transient (so $\tilde{V} h^{Y} \leqslant 1$ on $E_{\Delta}$ for some bounded Borel function $h^{Y}$ which is strictly positive on $E$ ) and that $\rho$ is a reference probability measure for $\tilde{Y}$ (so $\tilde{V}^{a}(x, \cdot) \ll \rho$ for all $x$ in $E$ and for all nonnegative $a$ ).

If we time change $\tilde{X}$ and $\tilde{Y}$ by the inverses of strictly increasing continuous additive functionals, then the hitting probabilities do not change. We shall find it convenient most of the time to work with the time changed versions which we now describe. Set $A_{t}=\int_{0}^{t} h^{X}\left(\tilde{X}_{s}\right) d s$ (resp. $\left.B_{t}=\int_{0}^{t} h^{Y}\left(\tilde{Y}_{s}\right) d s\right) ; A_{t}$ (resp. $B_{t}$ ) is a strictly increasing continuous additive functional of $\tilde{X}$ (resp. $\tilde{Y}$ ). If we define $T_{t}=$ $\inf \left\{s: A_{s}>t\right\}$, and if we set $\mathscr{F}=\tilde{\mathscr{F}}_{\mathcal{F}} \mathscr{F}_{t}=\tilde{\mathscr{F}}_{T(t)}, X_{t}=\tilde{X}_{T(t)}, \theta_{t}=\tilde{\theta}_{T(t)}, P^{x}=\tilde{P}^{x}$, then $X=\left(\Omega, \mathscr{F}, \mathscr{F}_{t}, X_{t}, \theta_{t}, P^{x}\right)$ is a right process on $\left(E_{\Delta}, \mathcal{E}_{\Delta}\right)$ with Borel measurable semigroup $P_{t}$ and resolvent $\left(U^{a}\right)_{a>0}$. Similarly, if we define $S_{t}=\inf \left\{s: B_{s}>t\right\}$, and if we set $\mathcal{G}=\tilde{\mathcal{G}}, \quad \mathcal{G}_{t}=\tilde{\mathcal{G}}_{S(t)}, \quad Y_{t}=\tilde{Y}_{S(t)}, \quad \Theta_{t}=\tilde{\theta}_{S(t)}, Q^{x}=\tilde{Q}^{x}$, then $Y=$ $\left(\Omega, \mathcal{G}, \mathcal{G}_{t}, Y_{t}, \Theta_{t}, Q^{x}\right)$ is a right process on $\left(E_{\Delta}, \mathcal{E}_{\Delta}\right)$ with Borel measurable semigroup $Q_{t}$ and resolvent $\left(V^{a}\right)_{a>0}$. Since $U 1=\tilde{U} h^{X} \leqslant 1$ on $E, \zeta=\inf \left\{t: X_{t}=\Delta\right\}<\infty$ almost surely $\left(P^{x}\right)$ for all $x$ in $E$. Since $V 1=\tilde{V} h^{Y} \leqslant 1$ on $E, z=\inf \left\{t: Y_{t}=\Delta\right\}<\infty$ almost surely $\left(Q^{x}\right)$ for all $x$ in $E$.

Reverse $X$ and $Y$ by setting

$$
\begin{aligned}
\hat{X}_{t}(\omega) & =X_{\zeta(\omega)-t}(\omega) & & \text { if } 0<t \leqslant \zeta(\omega), \\
& =\Delta & & \text { if } t>\zeta(\omega), \\
\hat{Y}_{t}(\omega) & =Y_{z(\omega)-t}(\omega) & & \text { if } 0<t \leqslant z(\omega), \\
& =\Delta & & \text { if } t>z(\omega) .
\end{aligned}
$$

Then $\left(\hat{X}_{t}, P^{\eta}\right)$ (resp. $\left.\left(\hat{Y}_{t}, Q^{\rho}\right)\right)$ is a left continuous moderate Markov process with a Borel semigroup and resolvent given by $\left(\hat{P}_{t}\right)$ and $\left(\hat{U}^{a}\right)\left(\right.$ resp. $\left(\hat{Q}_{t}\right)$ and $\left.\left(\hat{V}^{a}\right)\right)[3,10]$. The semigroups and resolvents may be chosen to have the following properties [12]. First, $\hat{U}^{a}(\cdot, x) \ll \lambda=\eta U$ and $\hat{V}^{a}(\cdot, x) \ll \xi=\rho V$ for all $a \geqslant 0$ and for all $x$ in $E$. Second, if we use the notation $\lambda(f)=\int f(x) \lambda(d x)$ and $\xi(f)=\int f(x) \xi(d x)$, then $\lambda\left(f \cdot U^{a} g\right)=\lambda\left(f \hat{U}^{a} \cdot g\right)$ and $\xi\left(f \cdot V^{a} g\right)=\xi\left(f \hat{V}^{a} \cdot g\right)$ for all $a \geqslant 0$ and for all positive Borel functions $f$ and $g$ on $E$. Here we use the convention that coresolvents and cosemigroups such as $\hat{U}^{a}$ and $\hat{P}_{t}$ act on functions on the left (see, for example, [1, Chapter VI]). Third, $\hat{P}_{t}$ and $\hat{Q}_{t}$ are moderate Markov semigroups. To explain this, let $W=\left\{w:(0, \infty) \rightarrow E_{\Delta}\right.$ such that $w(t)$ is left continuous and has trap $\left.\Delta\right\}$, let $\hat{X}_{t}(w)=\hat{Y}_{t}(w)=w(t)$, and let $\mathcal{W}^{0}=\sigma\left(\hat{X}_{s}: s \geqslant 0\right)$. For each $x$ in $E$, we can construct a measure $\hat{P}^{x}$ (resp. $\left.\hat{Q}^{x}\right)$ on $\left(W, \mathscr{Q} \int^{0}\right)$ so that if $0<t_{1}<t_{2}<\cdots<t_{n}$, and $\left(f_{i}\right)_{i \leqslant n} \subset b \mathcal{E}_{\Delta}^{+}$, then

$$
\hat{P}^{x}\left(f_{1}\left(\hat{X}_{t_{1}}\right) \cdots f_{n}\left(\hat{X}_{t_{n}}\right)\right)=f_{n} \hat{P}_{t_{n}-t_{n-1}} f_{n-1} \hat{P}_{t_{n-1}-t_{n-2}} f_{n-2} \cdots f_{1} \hat{P}_{t_{1}}(x)
$$

(resp. $\left.\hat{Q}^{x}\left(f_{1}\left(\hat{Y}_{t_{1}}\right) \cdots f_{n}\left(\hat{Y}_{t_{n}}\right)\right)=f_{n} \hat{Q}_{t_{n}-t_{n-1}} f_{n-1} \hat{Q}_{t_{n-1}-t_{n-2}} f_{n-2} \cdots f_{1} \hat{Q}_{t_{1}}(x)\right)$. The processes $\left(\hat{X}_{t}, \hat{P}^{x}\right)$ and $\left(\hat{Y}_{t}, \hat{Q}^{x}\right)$ have the Markov property at predictable times. If $f \hat{U}<\infty$ (resp. $f \hat{V}<\infty)$, then $f \hat{U}\left(\hat{X}_{t}\right)$ (resp. $f \hat{V}\left(\hat{Y}_{t}\right)$ ) is left continuous on $(0, \infty)$ almost surely. 
Thus $\left(U^{a}\right)$ and $\left(\hat{U}^{a}\right)$ are two resolvents in classical duality with respect to the finite excessive reference measure $\lambda$, and $\left(V^{a}\right)$ and $\left(\hat{V}^{a}\right)$ are in classical duality with respect to the finite excessive reference measure $\xi$. As in [1, Chapter VI], for each $a \geqslant 0$, we may choose potential densities $u^{a}(x, y) \in \varepsilon_{\Delta} \times \varepsilon_{\Delta}$ and $v^{a}(x, y) \in \varepsilon_{\Delta} \times \varepsilon_{\Delta}$ having the properties

$$
\begin{aligned}
& x \rightarrow u^{a}(x, y) \text { is } a \text {-excessive for }\left(U^{b}\right)_{b \geqslant 0} . \\
& x \rightarrow v^{a}(x, y) \text { is } a \text {-excessive for }\left(V^{b}\right)_{b \geqslant 0} . \\
& y \rightarrow u^{a}(x, y) \text { is } a \text {-excessive for }\left(\hat{U}^{b}\right)_{b \geqslant 0} . \\
& y \rightarrow v^{a}(x, y) \text { is } a \text {-excessive for }\left(\hat{V}^{b}\right)_{b \geqslant 0} .
\end{aligned}
$$

$$
U^{a} f(x)=\int u^{a}(x, y) f(y) \lambda(d y) ; \quad f \in \mathcal{E}_{\Delta}^{+}
$$

$$
V^{a} f(x)=\int v^{a}(x, y) f(y) \xi(d y) ; \quad f \in \varepsilon_{\Delta}^{+} .
$$

$$
f \hat{U}^{a}(y)=\int f(x) u^{a}(x, y) \lambda(d x) ; \quad f \in \mathcal{E}_{\Delta}^{+} .
$$

$$
f \hat{V}^{a}(y)=\int f(x) v^{a}(x, y) \xi(d x) ; \quad f \in \mathcal{E}_{\Delta}^{+} .
$$

For the time being, we restrict our attention to $X$ and $\hat{X}$, although to each assertion below corresponds an analogous assertion for $Y$ and $\hat{Y}$.

The fact that $\hat{X}$ is only moderately Markov and may not be a normal Markov process complicates matters a bit. One way used to overcome these complications has been to construct a compact metric space $\bar{E}_{\Delta}$ with Borel field $\overline{\mathcal{E}}_{\Delta}$ so that $\left(U^{a}\right)$ and $\left(\hat{U}^{a}\right)$ extend to be Ray resolvents on all of $\bar{E}_{\Delta}$ (which extensions we again denote by $U^{a}$ and $\left.\hat{U}^{a}\right), E_{\Delta} \in \overline{\mathcal{E}}_{\Delta}$, and $E_{\Delta}$ is dense in $\bar{E}_{\Delta}$. The compactification procedure is developed in [6]. A summary of these results and several complements are given in [8, §2], so we shall not repeat these results here. It will suffice for our purposes to note that $\hat{X}_{t+}=\lim _{s \downarrow \downarrow t} \hat{X}_{s}$ exists in the topology of $\bar{E}_{\Delta}$ almost surely $\left(\hat{P}^{x}\right)$ for all $x$ in $E$, and that $\hat{X}_{t+}$ is a right continuous strong Markov process. Let $\hat{D}=\left\{x \in \bar{E}_{\Delta}\right.$ : $\lim _{a \rightarrow \infty} a f \hat{U}^{a}(x)=f(x)$ for all $\left.f \in b \mathbf{C}\left(\bar{E}_{\Delta}\right)^{+}\right\}$, and let $\hat{C}=\hat{D} \cap E$. Then $\left(\hat{U}^{a}\right)$ restricted to $\hat{D}$ is the resolvent of a right process on $\hat{D}$. If $x \in \hat{C}$, then $\left(\hat{X}_{t+}, \hat{P}^{x}\right)$ is a realization of this process. Since $\bar{E}-\hat{D}$ are branch points, $\hat{X}_{t}$ visits $\bar{E}-\hat{D}$ only countably often, and $\lambda(\bar{E}-\hat{D})=0$.

We now present two results to be used in the proof of the main Theorem (1.3).

(1.1) ThEOREM. Let $A_{t}$ be a predictable additive functional of $\hat{X}_{t}$ with a bounded potential which does not charge $\hat{\zeta}=\inf \left\{t: \hat{X}_{t}=\Delta\right\}$. There is a measure $\nu$ on $\left(E_{\Delta}, \varepsilon_{\Delta}\right)$ so that for each $f \in b \mathcal{E}_{\Delta}^{+}$, and for each $x$ in $E$,

$$
\hat{P}^{x} \int f\left(\hat{X}_{s^{-}}\right) d A_{s}=\int f(y) u(y, x) \nu(d y)=\nu f \hat{U}(x) .
$$


Proof. The measure $\nu$ is the Revuz measure of $A_{t}$ defined by setting $\nu(g)=$ $\lim _{a \rightarrow \infty} a \hat{P}^{\lambda} \int e^{-a s} g\left(\hat{X}_{s-}\right) d A_{s}$, for each $g \in b{\mathcal{E}_{\Delta}^{+}}^{+}$. Since $\hat{P}^{\lambda}\left(\hat{X}_{s-} \in \bar{E}_{\Delta}-E_{\Delta}\right.$ for some $s>0)=0, \nu\left(\bar{E}_{\Delta}-E_{\Delta}\right)=0$. Since $\hat{X}_{s+}$ has a strong Markov dual (namely $X$ ) on $\bar{E}_{\Delta}$, the rest of the proof follows as in Revuz [11] (see also [6 and 9]). Q.E.D.

It is interesting to note that in order to represent potentials of additive functionals of $X$, one must allow $\nu$ to charge $\bar{E}_{\Delta}-E_{\Delta}$ in general. In this respect, $\hat{X}_{t}$ is better behaved than $X$.

(1.2) Corollary. If $f \hat{U}(x)$ and $g \hat{U}(x)$ are bounded, and $f$ and $g$ are positive, then there is a measure $\nu$ on $E$ so that $f \hat{U}(x) \wedge g \hat{U}(x)=\nu \hat{U}(x)$ for all $x$ in $\hat{C}$.

Proof. Since $f \hat{U}(x)$ and $g \hat{U}(x)$ are excessive functions for the resolvent $\left(\hat{U}^{a}\right)$ on $\hat{D}$ (which is the resolvent of a right process), $p(x)=f \hat{U}(x) \wedge g \hat{U}(x)$ is excessive for the process $\hat{X}_{t+}$ restricted to $\hat{D}$. But $p\left(\hat{X}_{t+}\right)$ is a right continuous supermartingale dominated by the potential $f \hat{U}\left(\hat{X}_{t+}\right)$; so there is a predictable additive functional $A_{t}$ not charging $\hat{\zeta}$ with $p(x)=\hat{P}^{x}\left(A_{\infty}\right)$ for all $x$ in $\hat{D}$. The proof is finished by applying Thereom (1.1). Q.E.D.

(1.3) THEOREM. Let $\tilde{X}$ and $\tilde{Y}$ be two transient right processes on $\left(E_{\Delta}, \varepsilon_{\Delta}\right)$, each possessing a reference measure. Assume

(1.4) for each compact set $L \subset E, \tilde{P}^{x}\left(T_{L}<\infty\right)=\tilde{Q}^{x}\left(T_{L}<\infty\right)$;

(1.5) within each nonpolar set $G \subset E$, there is a nonpolar compact set $L \subset G$ so that $\tilde{P}^{x}\left(T_{L}<\infty\right)=U \pi_{L}(x)$ for some measure $\pi_{L}$ supported on $L$ and $\tilde{Q}^{x}\left(T_{L}<\infty\right)=$ $V \gamma_{L}(x)$ for some measure $\gamma_{L}$ supported on $L$.

There is a continuous additive functional $H_{t}$ of $\tilde{Y}$ which is strictly increasing and finite up to the lifetime of $\tilde{Y}$ so that if we set $\tau_{t}=\inf \left\{s: H_{s}>t\right\}$, then $\left(\tilde{X}_{t}, \tilde{P}^{x}\right)$ has the same law as that of the right process $\left(\tilde{Y}_{\tau(t)}, \tilde{Q}^{x}\right)$.

Since (1.4) implies that the polar sets of $\tilde{X}$ are the same as the polar sets of $\tilde{Y}$, it makes sense to talk about "nonpolar sets" in (1.5) without specifying the process. Recall that $T_{L}$ is defined to be the infimum of the strictly positive times that the process is in $L$.

The rest of this section is devoted to the proof of Theorem (1.3). Clearly, we shall have to work extensively with potentials of measures, and it is therefore natural to introduce a topology generated by a class of these potentials. We do this below, and the reader will notice a certain similarity with the Ray-Knight compactification procedure. This compactification is simpler, however.

(1.6) LEMMA. There is a Borel function $h$ on $\bar{E}$ which is strictly positive and bounded on $\hat{D}$ so that $h \hat{U}(x) \leqslant 1$ for all $x$ in $\bar{E}$.

Proof. Since $\infty>\lambda(U 1)=\lambda(1 \hat{U}), 1 \hat{U}(x)<\infty$ except on some polar set $\hat{\Gamma}$. Therefore, $\hat{X}_{t+}$ restricted to $\hat{D}-\hat{\Gamma}$ has $1 \hat{U}<\infty$. By [5, Proposition (2.2)], there is a strictly positive bounded function $h$ so that $h \hat{U} \leqslant 1$ on $\hat{D}-\hat{\Gamma}$. Notice that $h$ may be chosen to be Borel measurable since every excessive function is Borel (thanks to the existence of a reference measure). Therefore, $h \hat{U}$ is bounded by 1 on $\hat{D}$, and hence on all of $\bar{E}_{\Delta}$. Q.E.D. 
The constant function 2 is excessive for $\left(\hat{U}^{a}\right)$ restricted to $\hat{D}$, so we can find bounded positive Borel functions $\left(g_{n}\right)_{n \geqslant 1}$ which are $\lambda$-integrable so that $g_{n} \hat{U}$ increases to 2 on $\hat{D}$. Let

$$
\mathbf{S}^{+}=\left\{\sum_{k=1}^{n}\left(h_{k} h+c_{k} g_{k}\right) \hat{U}(x):\left(h_{k}\right) \subset \mathbf{C}\left(\bar{E}_{\Delta}\right)^{+}, c_{k} \geqslant 0, n \geqslant 1\right\} .
$$

Then $\mathbf{S}^{+}$is separable in the uniform norm since $h \hat{U} \leqslant 1$. Set

$$
\mathbf{R}^{+}=\left\{\sum_{k=1}^{n} a_{k} h_{1}^{k} \wedge \cdots \wedge h_{n_{k}}^{k} \wedge c_{k}:\left(h_{j}^{k}\right) \subset \mathbf{S}^{+}, a_{k} \geqslant 0, c_{k} \geqslant 0, n_{k} \geqslant 1\right\} .
$$

Then $\mathbf{R}=\mathbf{R}^{+}-\mathbf{R}^{+}$is also separable in the uniform norm. Using the identity $(a-b) \wedge(c-d)=(a+d) \wedge(c+b)-(d+b)$, one can check that $\mathbf{R}$ is a vector lattice. By [1, Proposition (V5.10)], $\hat{U}^{a}$ can be reconstructed from knowledge of $\left\{g \hat{U}: g \in \mathbf{C}\left(\bar{E}_{\Delta}\right)^{+}\right\}$, so $\mathbf{R}$ must separate points on $\hat{C}$.

Let $F=\prod_{k=1}^{\infty}[0,1]$, let $\left(h^{i}\right)$ be a sequence in $\mathbf{R}$ which is dense in $\mathbf{R}$ in the uniform norm, and let $\Phi: \hat{C} \rightarrow F$ by setting $\Phi(x)=\left(h^{i}(x) /\left\|h^{i}\right\|_{\infty}\right)_{i \geqslant 1}$. We take the metric $d$ on $\hat{C}$ induced by a metric on $F$ compatible with the product topology of $F$ and given by

$$
d(x, y)=\sum_{i=1}^{\infty} 2^{-i} \frac{\left|h^{i}(x)-h^{i}(y)\right|}{1+\left|h^{i}(x)-h^{i}(y)\right|} .
$$

Close $\hat{C}$ in this metric to obtain a compact metric space $\bar{C}$. Each function $g$ in $\mathbf{R}$ may be extended to a continuous function $\bar{g}$ on $\bar{C}$. Let $\overline{\mathbf{R}}=\{\bar{g}: g \in \mathbf{R}\}$. Since $\overline{\mathbf{R}}$ may not contain the constant functions, we cannot say that $\overline{\mathbf{R}}$ is dense in $\mathbf{C}(\bar{C})$. But if we let $\bar{C}_{n}$ be the closure in $\bar{C}$ of $\hat{C}_{n}=\left\{x \in \hat{C}: g_{n} \hat{U}(x)>1\right\}$, then $\overline{\mathbf{R}}\left(\bar{C}_{n}\right)$ (defined to be the restrictions to $\bar{C}_{n}$ of functions in $\left.\overline{\mathbf{R}}\right)$ contains the constant functions on $\bar{C}_{n}$. By the vector-lattice form of the Stone-Weierstrass theorem, $\overline{\mathbf{R}}\left(\bar{C}_{n}\right)$ is dense in $\mathbf{C}\left(\bar{C}_{n}\right)$.

(1.7) Proposition. To each $f \in \mathbf{R}$, there corresponds a finite signed measure $\nu$ on $E$ so that $f(x)=\nu \hat{U}(x)$ for all $x$ in $\hat{C}$.

Proof. Every function $f \in \mathbf{S}$ satisfies the proposition by the construction of $\mathbf{S}$. To complete the proof, we need only show that if $f \hat{U}(x) \in \mathbf{S}^{+}$and $g \hat{U}(x) \in \mathbf{S}^{+}$, then $f \hat{U}(x) \wedge g \hat{U}(x)$ satisfies the proposition. By (1.2), $f \hat{U}(x) \wedge g \hat{U}(x)=\nu \hat{U}(x)$ on $\hat{C}$ for some positive measure $\nu$ on $E$. We may find a sequence of positive functions $\left(\phi_{n}\right)$ so that $U \phi_{n}(x)$ increases to 1 on $E$. Therefore,

$$
\begin{aligned}
\infty & >\lambda(g)=\lim _{k \rightarrow \infty} \int g \hat{U}(x) \phi_{k}(x) \lambda(d x) \\
& \geqslant \lim _{k \rightarrow \infty} \int \nu \hat{U}(x) \phi_{k}(x) \lambda(d x) \\
& =\nu(E) . \quad \text { Q.E.D. }
\end{aligned}
$$

Since $g_{n} \hat{U}(x)$ increases to 2 on $\hat{C}, \hat{C}_{n}$ increases to $\hat{C}$, and it will suffice to fix $n$ and to work with $\hat{C}_{n}$. Given any closed set $\bar{K} \subset \bar{C}_{n}$, there is a bounded sequence of functions $\left(\bar{\phi}_{k}\right) \subset \mathbf{C}\left(\bar{C}_{n}\right)^{+}$so that $\bar{\phi}_{k}$ decreases to $1_{\bar{K}}$. For each $k$, choose $\bar{h}_{k} \in \overline{\mathbf{R}}$ so 
that $\sup \left\{\left|\bar{h}_{k}(x)-\bar{\phi}_{k}(x)\right|: x \in \bar{C}_{n}\right\}<1 / k^{2}$. Then $\bar{h}_{k}+\left(1 / k^{2}\right) \overline{g_{n} \hat{U}}(x)$ is positive and converges boundedly to $1_{K}$ on $\bar{C}_{n}$. Let $K=\bar{K} \cap \hat{C}_{n}$. Since $h_{k}+\left(1 / k^{2}\right) g_{n} \hat{U}(x) \in$ $\mathbf{R}$ is of the form $\mu_{k} \hat{U}(x)$ for all $x$ in $\hat{C}, \mu_{k} \hat{U}(x) \cdot 1_{\hat{C}_{n}}(x)$ converges boundedly to $1_{K}(x)$ as $k$ increases to infinity. Let $L$ be any compact subset contained in $\hat{C}_{n}$ satisfying (1.5). (Important note: "compact subset" without reference to a topology means "compact in the original topology of $E$ ".) So $P^{x}\left(T_{L}<\infty\right)=U \pi_{L}(x)$ with the support of $\pi_{L}$ contained in $L$, and $P^{\mu(k)}\left(T_{L}<\infty\right)$ converges to $\pi_{L}(K)$ by the Lebesgue dominated convergence theorem. By (1.4), $P^{\mu(k)}\left(T_{L}<\infty\right)=$ $Q^{\mu(k)}\left(T_{L}<\infty\right)$ (recall that $\mu_{k}$ is a finite signed measure, so the equality makes sense). If we use the notation $\mu_{k} U \pi_{L}$ to denote $\int U \pi_{L}(x) \mu_{k}(d x)$, then we have that $\mu_{k} U \pi_{L}=\mu_{k} V \gamma_{L}$ converges to $\pi_{L}(K)$.

(1.8) LEMMA. $\mu_{k} \hat{V}(x)$ is well defined on $\hat{C}_{n}$ except possibly on a polar set $\Gamma_{k}$.

Proof. Since $\mu_{k}^{+} U \pi_{L}<\infty$ and $\mu_{k}^{-} U \pi_{L}<\infty$, Fubini's theorem guarantees that $\mu_{k}^{+} \hat{V}(x)$ and $\mu_{k}^{-} \hat{V}(x)$ are finite almost surely $\left(\gamma_{L}\right)$ for every compact subset $L$ of $\hat{C}_{n}$ satisfying (1.5). Therefore, $\mu_{k}^{+} \hat{V}(x)$ and $\mu_{k}^{-} \hat{V}(x)$ are finite everywhere on $\hat{C}_{n}$ except perhaps on a polar set $\Gamma_{k}$, so $\mu_{k} \hat{V}(x)=\mu_{k}^{+} \hat{V}(x)-\mu_{k}^{-} \hat{V}(x)$ is well defined except on $\Gamma_{k}$. Q.E.D.

We set $\Gamma=\cup_{k} \Gamma_{k}$. Notice that $\Gamma$ depends on the sequence $\left(\mu_{k}\right)$.

(1.9) Proposition. $\mu_{k} \hat{V}(x)$ converges boundedly on $\hat{C}_{n}-\{$ a polar set depending on $\left.\left(\mu_{k}\right)\right\}$ to a function $f^{K}$ supported on $K \cup\left\{\right.$ a polar set depending on $\left.\left(\mu_{k}\right)\right\}$. Moreover, $\left\{\mu_{k} \hat{V}(x)<0\right\} \cap\left(\hat{C}_{n}-\Gamma\right)$ is polar.

Proof. To prove the last statement, let $L$ be any compact subset of $\left\{\mu_{k} \hat{V}(x)<0\right\}$ $\cap\left(\hat{C}_{n}-\Gamma\right)$ satisfying (1.5). Since $0 \leqslant \mu_{k} U \pi_{L}=\mu_{k} V \gamma_{L} \leqslant 0$, we conclude $\pi_{L}=\gamma_{L}=$ 0 , so $L$ must be polar. Now let $G_{k}=\left\{x: \mu_{k} \hat{V}(x)+\left(2 / k^{2}\right) g_{n} \hat{V}(x)<\mu_{k+1} \hat{V}(x)\right\} \cap$ $\left(\hat{C}_{n}-\Gamma\right)$. Choose a compact subset $L \subset G_{k}$ satisfying (1.5) so that $\mu_{k} V \gamma_{L}+$ $\left(2 / k^{2}\right) g_{n} V \gamma_{L}<\mu_{k+1} V \gamma_{L}$. By (1.4) and (1.5), we have that $\mu_{k} U \pi_{L}+\left(2 / k^{2}\right) g_{n} U \pi_{L}<$ $\mu_{k+1} U \pi_{L}$. But by our choice of the $\left(\mu_{k}\right), \mu_{k} \hat{U}(x)+\left(2 / k^{2}\right) g_{n} \hat{U}(x)>\mu_{k+1} \hat{U}(x)$ on $\hat{C}_{n}$. Therefore, $\pi_{L}=\gamma_{L}=0$ and $G_{k}$ must be polar. Thus if we set $E_{n}=\left(\cup_{k} G_{k} \cup \Gamma\right)^{c}$ $\cap \hat{C}_{n}, \mu_{k} \hat{V}(x)+\left(2 / k^{2}\right) g_{n} \hat{V}(x)>\mu_{k+1} \hat{V}(x)$ on $E_{n}$, and we conclude that $\mu_{k} \hat{V}(x)$ converges to some function $f^{K}(x)$ on $E_{n}$. Moreover, $0 \leqslant \mu_{k} \hat{V}(x) \leqslant \mu_{1} \hat{V}(x)+$ $2\left(\sum k^{-2}\right) g_{n} \hat{V}(x)$ on $C_{n}$. To examine the support of $f^{K}$, let $L$ be any compact subset of $\hat{C}_{n}-K$ satisfying (1.5). Then $0=\pi_{L}(K)=\lim _{k \rightarrow \infty} \mu_{k} U \pi_{L}=\lim _{k \rightarrow \infty} \mu_{k} V \gamma_{L}=$ $\gamma_{L}\left(f^{K}\right)$, the last equality holding by the dominated convergence theorem. Q.E.D.

(1.10) Proposition. If $\bar{J} \subset \bar{K} \subset \bar{C}_{n}$ are compact sets in the topology of $\bar{E}_{\Delta}$, then $\{x$ : $\left.f^{K}(x) \neq f^{J}(x)\right\} \cap J$ is a polar set.

Proof. Let $L$ be any compact subset of $M=\left\{f^{K}>f^{J}\right\} \cap J$ satisfying (1.5). Then $\gamma_{L}\left(f^{J}\right)=\pi_{L}(J)=\pi_{L}(K)=\gamma_{L}\left(f^{K}\right)$, so $L$ must be polar. Thus $M$ is polar, and the case $N=\left\{f^{K}<f^{J}\right\} \cap J$ is similar. Q.E.D.

(1.11) Proposition. Let $f^{n}=f^{\hat{C}_{n}}$. If $L$ is any compact subset of $\hat{C}_{n}$ satisfying (1.5), then $\pi_{L}(B)=\gamma_{L}\left(1_{B} f^{n}\right)$ for all Borel sets $B \subset \hat{C}_{n}$. 
Proof. If $B \in \mathcal{E}$, then $B \in \overline{\mathcal{E}}$. By inner regularity of the measure $\pi_{L}, \pi_{L}(B)=$ $\sup \left\{\pi_{L}(\bar{K})=\pi_{L}(K): \bar{K}\right.$ is compact in $\left.\bar{E}, K \subset B\right\}$. By $(1.10), \gamma_{L}\left(f^{K}\right)=\gamma_{L}\left(1_{K} f^{n}\right)$, and $\sup \left\{\gamma_{L}\left(1_{K} f^{n}\right): K\right.$ is compact in $\left.E, B \supset K\right\}=\gamma_{L}\left(1_{B} f^{n}\right)$ by inner regularity of $\gamma_{L}$. Q.E.D.

Thus if $L$ is any compact subset of $\hat{C}_{n}$ satisfying (1.5), then $\int u(x, y) \pi_{L}(d y)=$ $\int u(x, y) f^{n}(y) \gamma_{L}(d y)$ for all $x$. So for each fixed $x, \Lambda(n, x)=\left\{y \in \hat{C}_{n}: v(x, y) \neq\right.$ $\left.u(x, y) f^{n}(y)\right\}$ is a polar set. It is easy to check that $\left\{y: f^{n+j}(y) \neq f^{n}(y)\right\} \cap \hat{C}^{n}$ is polar, so there is a function $f$ defined on all of $\hat{C}$ so that $\Lambda(x)=\{y \in \hat{C}$ : $v(x, y) \neq u(x, y) f(y)\}$ is a polar set for each $x$. It follows from (1.11) that $\{y \in \hat{C}$ : $f(y)=0\}$ is a polar set, so $f^{-1}(y)$ makes sense. Now $\lambda$ does not charge $E-\hat{C}$, so $U g(x)=\int u(x, y) f(y)\left[f^{-1}(y) g(y) \lambda(d y)\right]=\int v(x, y) f^{-1}(y) g(y) \lambda(d y)$, which shows that the cone of excessive functions for $X, S(X)$, is contained in the cone of excessive functions for $Y, S(Y)$. As we mentioned before Theorem (1.1), all of the preceding arguments apply equally well to $Y$. Doing so, we have the following results. Let $E_{\Delta}^{Y}$ be the appropriate compactification for $\left(V^{a}\right)$ and $\left(\hat{V}^{a}\right)$, and let $\hat{C}(Y)=\left\{x \in E: \lim _{a \rightarrow \infty} a g \hat{V}^{a}(x)=g(x)\right.$ for all $\left.g \in b \mathbf{C}\left(\bar{E}_{\Delta}^{Y}\right)^{+}\right\}$. There is an extension of $f$ to $\hat{C} \cup \hat{C}(Y)$ (which we again denote by $f$ ) so that $\{y \in \hat{C} \cup C(Y)$ : $v(x, y) \neq u(x, y) f(y)\}$ is a polar set for each $x$. Moreover, $S(Y) \subseteq S(X)$, so we conclude that $S(X)=S(Y)$. Hunt's balayage theorem [1, p. 141] now guarantees that $X$ and $Y$ have the same hitting distributions: $P_{K}(x, \cdot)=Q_{K}(x, \cdot)$. At this point, we might quickly apply the Blumenthal-Getoor-McKean Theorem to finish the proof, but we do not need to use this result. The function $U 1$ is a bounded excessive function for $Y$ which is the potential of a continuous additive functional of $Y$ by [1, IV-3.8]. Let $Z_{t}$ denote this additive functional.

(1.12) Proposition. $Z_{t}$ is strictly increasing.

Proof. If we let $R=\inf \{t: Z(t)>0\}$, then $I=\left\{x: Q^{x}(\exp (-R))=1\right\}$ is the fine support of $Z_{t}$, and we need to show that $I=E$. If $I \neq E, I^{c}$ is finely open (for $X$ and for $Y$ ), and we choose a point $x$ in the complement of $I$. Then $U 1(x)>$ $P_{I} U 1(x)=Q_{I} U 1(x)$. Thus $x$ cannot be in $I^{c}$. Q.E.D.

Let $\sigma(t)=\inf \{s: Z(s)>t\}$. If $g \in b{\varepsilon_{\Delta}^{+}}^{+}$, then $Q^{x} \int g\left(Y_{\sigma(t)}\right) d t=Q^{x} \int g\left(Y_{t}\right) d Z(t)$ $=\int v(x, y) g(y) \nu(d y)=\int u(x, y) g(y) f(y) \nu(d y)$, where $\nu$ is the Revuz measure of $Z(t)$ defined by setting $\nu(g)=\lim _{a \rightarrow \infty} a Q^{\xi} \int e^{-a t} g\left(Y_{t-}\right) d Z(t)$. Recall that if $m_{1}$ and $m_{2}$ are Revuz measures of continuous additive functionals with bounded potentials and $U m_{1}(x)=U m_{2}(x)$ for all $x$ in $E$ then $m_{1}=m_{2}$. Since $Q^{x}(Z(\infty))=U 1(x)=$ $\int u(x, y) \lambda(d y)$, we conclude that $\nu(d y)=f^{-1}(y) \lambda(d y)$. If we define $V_{Z}^{a} g(x)=$ $Q^{x} \int e^{-a t} g\left(Y_{\sigma(t)}\right) d t$ for all $a \geqslant 0$, then $V_{Z}=U$. By [1, V-5.10], it follows that $V_{Z}^{a}=U^{a}$ for all $a \geqslant 0$. Therefore, $\left(X_{t}, P^{x}\right)$ has the same law as $\left(Y_{\sigma(t)}, Q^{x}\right)$. Since $X_{t}$ (resp. $\left.Y_{t}\right)$ is a time change of $\tilde{X}_{t}$ (resp. $\tilde{Y}_{t}$ ) by the inverse of a strictly increasing continuous additive functional, the conclusion of Theorem (1.3) follows. Q.E.D.

2. Hunt processes, standard processes,.... We say that a right process $\tilde{X}$ is a Hunt process if $\tilde{X}$ is quasi-left continuous. That is, whenever $(T(n))$ is an increasing sequence of $\left(\tilde{\mathscr{F}}_{t}\right)$-optional times with limit $T$, then $\tilde{X}(T(n))$ converges to $\tilde{X}(T)$ on $\{T<\infty\}$. 
(2.1) THEOREM. Let $\tilde{X}$ and $\tilde{Y}$ be two transient Hunt processes on $\left(E_{\Delta}, \varepsilon_{\Delta}\right)$, each possessing a reference measure. Assume that $\tilde{P}^{x}\left(T_{L}<\infty\right)=\tilde{Q}^{x}\left(T_{L}<\infty\right)$ for each compact set $L \subseteq E$. There is a continuous additive functional $H(t)$ of $\tilde{Y}$ which is strictly increasing and finite up to the lifetime of $\tilde{Y}$ so that if we set $\tau(t)=\inf \{s: H(s)>t\}$, then $\left(\tilde{X}_{t}, \tilde{P}^{x}\right)$ has the same law as that of the process $\left(\tilde{Y}_{\tau(t)}, \tilde{Q}^{x}\right)$.

Proof. We proved in [8] that if $M=\sup \{t: \tilde{X}(t) \in L\}<\infty$ almost surely, then $\tilde{P}^{x}\left(f\left(\tilde{X}_{M}\right) ; M>0\right)=\int u(x, y)\left(1_{L} f(y) \kappa(d y)+f(z) \nu(d y, d z)\right)$, where $\kappa$ is a measure on $E$ and $\nu$ is a measure on $E \times E$ so that $\nu((E-L) \times E)=0$. Given a nonpolar set $G \subset E$, we can find a nonpolar compact set $L \subset G$ so that $M=$ $\sup \{t: \tilde{X}(t) \in L\}<\infty$ almost surely and so that $N=\sup \{t: \tilde{Y}(t) \in L\}<\infty$ almost surely. By the result cited above, $\tilde{P}^{x}\left(T_{L}<\infty\right)=U \pi_{L}(x)$ and $\tilde{Q}^{x}\left(T_{L}<\infty\right)=$ $V \gamma_{L}(x)$, where $\pi_{L}$ and $\gamma_{L}$ are measures supported on $L$. Therefore, hypothesis (1.5) is verified, and Theorem (1.3) applies here. Q.E.D.

Suppose now that $\tilde{X}$ and $\tilde{Y}$ are simply right processes. Can (1.5) be verified? The usual Ray-Knight procedure [4] produces two compact metric spaces $F(X)$ and $F(Y)$ so that $E \subset F(X)$ and $E \subset F(Y)$. Considered as a process on $F(X)$ (resp. $F(Y)$ ), $\tilde{X}$ (resp. $\tilde{Y}$ ) is a right process when restricted to the nonbranch points. A set $L \subset E$ is $X$-Ray-closed (resp. $Y$-Ray-closed) if $L$ is closed in $F$. It is true [8, Theorem (27)] that if $L$ is a transient $X$-Ray-closed set, then there is a measure $\pi_{L}$ supported on $L$ so that $\tilde{P}^{x}\left(T_{L}<\infty\right)=U \pi_{L}(x)$. Similarly, if $L$ is a transient $Y$-Ray-closed set, then there is a measure $\gamma_{L}$ supported on $L$ so that $\tilde{Q}^{x}\left(T_{L}<\infty\right)=V \gamma_{L}(x)$. It is not really important in (1.5) that $L$ be compact in the original topology (we did not use the compactness anywhere). The crucial question here is: given $G$ nonpolar, can we always choose $L \subset G$ so that $L$ is simultaneously $X$-Ray-closed and $Y$-Ray-closed? (We conjecture that the answer is yes.) To decide this question, it might be useful to have some sort of Ray compactification based on hitting probabilities!? This question we leave open.

With some slight additional regularity hypotheses, (1.5) can be verified. For example, let $\mathcal{K}=\left\{f \in b \mathcal{E}_{\Delta}^{+}: f \tilde{U}\left(X_{t_{-}}\right)\right.$be left continuous almost surely on $\left.(0, \infty)\right\}$, and assume that $\mathcal{H}$ is rich enough to guarantee that $m_{1}=m_{2}$ whenever $m_{1}(f)=$ $m_{2}(f)$ for all $f$ in $\mathcal{H}$. These are conditions given by Meyer [9] which ensure that the representation theory of Revuz holds true. In this case, if $A(t)$ is a predictable additive functional with bounded potential and Revuz measure $m$, then $\tilde{P}^{x}(A(\infty))$ $=\int u(x, y) m(d y)$. Standard processes with duals as discussed in [1, Chapter VI] are well-known examples of processes satisfying these assumptions.

\section{REFERENCES}

1. R. M. Blumenthal and R. K. Getoor, Markov processes and potential theory, Academic Press, New York, 1968.

2. R. M. Blumenthal, R. K. Getoor and H. P. McKean, Jr., Markov processes with identical hitting distributions, Illinois J. Math. 6 (1962), 402-420.

3. K. L. Chung and J. B. Walsh, To reverse a Markov process, Acta Math. 123 (1970), 225-251.

4. R. K. Getoor, Markov processes: Ray processes and right processes, Lecture Notes in Math., vol. 440, Springer-Verlag, Berlin, Heidelberg and New York, 1975.

5. __. Transience and recurrence of Markow processes, Lecture Notes in Math., vol. 784, Springer-Verlag, Berlin, Heidelberg and New York, 1980, pp. 397-409. 
6. J. Glover, Compactifications for dual processes, Ann. Probab. 8 (1980), 1119-1134.

7. __ Markov processes with identical last exit distributions, Z. Wahrsch. Verw. Gebiete 59 (1982), $67-75$.

8. __ Representing last exit potentials as potentials of measures, $\mathrm{Z}$. Wahrsch. Verw. Gebiete (to appear).

9. P. A. Meyer, Note sur l'interpretation des mesures d'equilibre, Lecture Notes in Math., vol. 321, Springer-Verlag, Berlin, Heidelberg and New York, 1973, pp. 210-216.

10. Le retournement du temps d'apres Chung et Walsh, Lecture Notes in Math., vol. 191, Springer-Verlag, Berlin, Heidelberg and New York, 1971, pp. 213-236.

11. D. Revuz, Mesures associes aux fonctionelles additives de Markov. I, Trans. Amer. Math. Soc. 148 (1970), 501-531.

12. R. Smythe and J. B. Walsh, The existence of dual processes, Invent. Math. 19 (1973), 113-148.

DEPARTMENT OF MATHEMATICS, UNIVERSITY OF Rochester, ROCHESTER, NEW YoRK 14627

Current address: Department of Mathematics, C-012, University of California, La Jolla, California 92093 